\title{
Introducción de LinkedIn en la gestión del deporte para crear entornos de aprendizaje en línea
}

Samuel López-Carril ${ }^{\mathrm{a}}$, María Huertas González-Serrano ${ }^{\mathrm{b}}$ y Paloma Escamilla-Fajardoc

aUniversitat de València, Departamento de Educación Física y Deportiva, samuel.lopez@uv.es, buniversitat de València, Departamento de Educación Física y Deportiva, m.huertas.gonzalez@uv.es y cUniversitat de València, Departamento de Educación Física y Deportiva, paloma.escamilla@uv.es

\section{\$EWWDW}

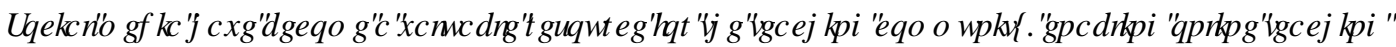
DQCE ODDQQD

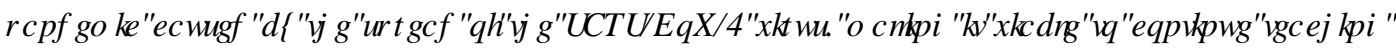

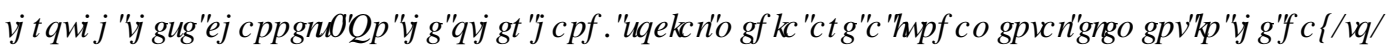

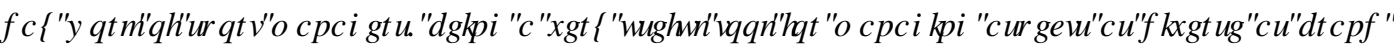

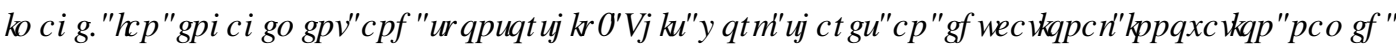

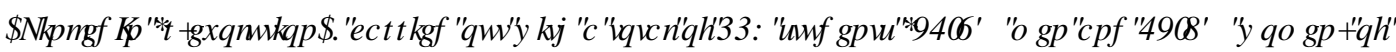

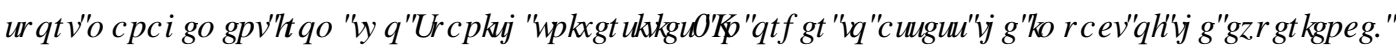

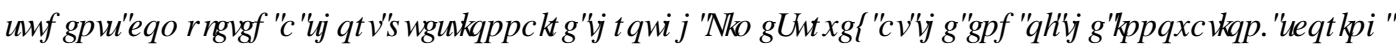

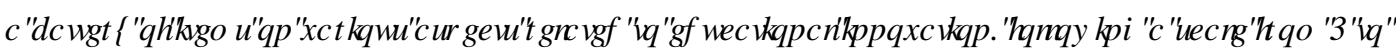

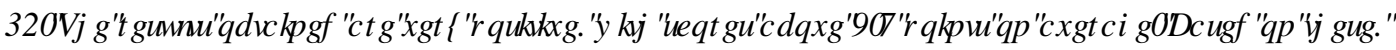

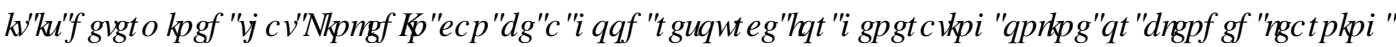
HQYILQP HQWD

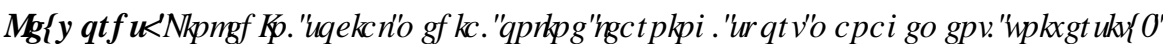

प

\section{HXP HQ}

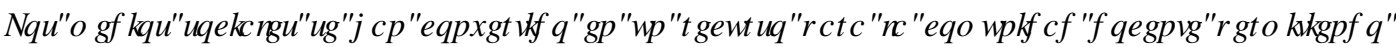

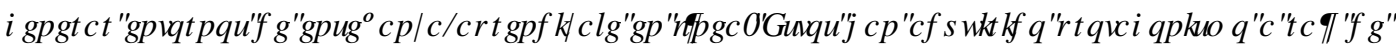

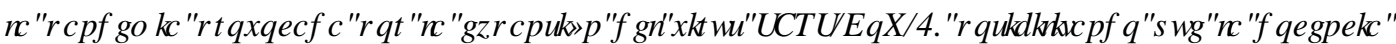

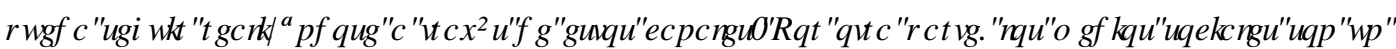

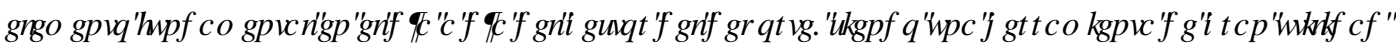

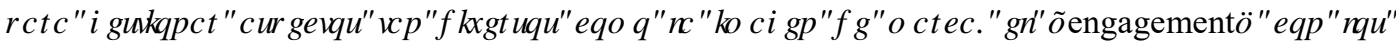

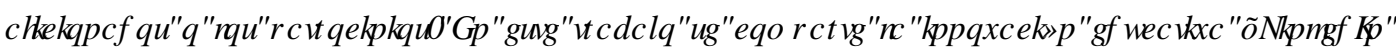

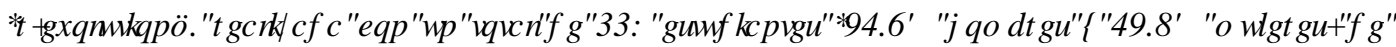

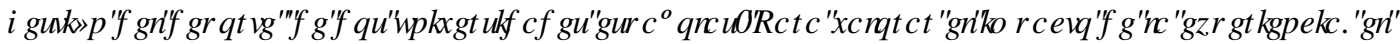

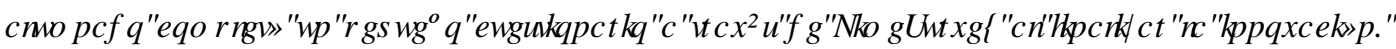

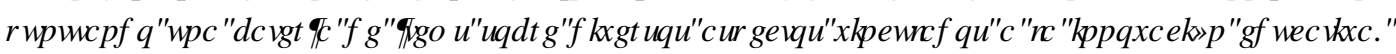

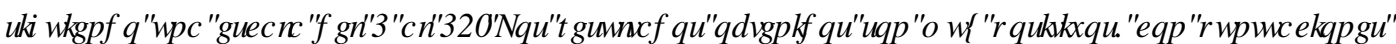

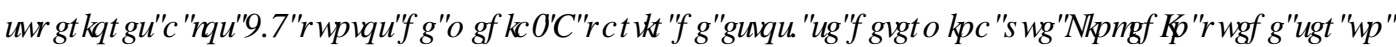
EXHQUFXURISDDJHQHDUHSDFIRVGHDSUHQC] DNAIHQQQHDRP L[ URVW

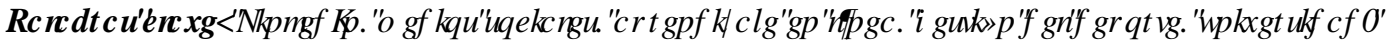




\section{Introducción}

En un contexto donde el proceso de digitalización y los avances tecnológicos hacen que los seres humanos pasen cada vez un mayor tiempo en línea (Kolokytha et al., 2015), los medios sociales, término que según Kaplan y Haenlein (2010, p. 61) engloba al "grupo de aplicaciones basadas en Internet que se apoyan en los fundamentos ideológicos y tecnológicos de la Web 2.0 y que permiten la creación y el intercambio de contenidos generados por los usuarios", se han convertido en un elemento central de la vida cotidiana tanto en el ámbito profesional como en el privado (Selwyn y Stirlinedg, 2016). De hecho, según Statista (2020), en 2025 , se espera que 4400 millones de personas sean usuarios de medios sociales, una cifra que supera más de la mitad de la población mundial.

La industria del deporte no ha sido ajena al auge de los medios sociales (Abeza et al., 2015), contribuyendo a transformar y a remodelar tanto el sistema de producción como el consumo del producto deportivo (Li et al., 2019; Yan et al., 2019). De hecho, los medios sociales han cambiado la forma en la que los consumidores se relacionan con las marcas y las empresas del sector deportivo (Chmait et al., 2020). Cada día, entrenadores, clubes, federaciones, deportistas, aficionados, y otros actores del deporte, utilizan estas herramientas comunicativas para interactuar (López-Carril et al., 2019). Por ello, los medios sociales se han convertido en un elemento muy importante para los gestores del deporte (Filo et al., 2015), pudiendo ser utilizados para favorecer el HDDJPHQnde los aficionados (Anagnostopoulos et al., 2018), así como una herramienta de PDUMMJ para construir la imagen de marca de una entidad o producto deportivo (Parganas et al., 2017).

Por otra parte, el sector de la educación también se ha visto influenciado por el creciente rol que los medios sociales tienen en la sociedad (Manca, 2020), transformando las prácticas docentes y contribuyendo en la creación de nuevos entornos de enseñanza-aprendizaje (Komljenovic, 2019). Este proceso se ha visto recientemente impulsado por los efectos que el virus SARS-CoV-2 ha provocado en el mundo (Ratten y Jones, 2020), causando una pandemia que ha llevado a que las universidades y escuelas se hayan visto afectadas por períodos de cierre o de acceso restringido como medida para frenar el avance del virus (Dwidienawati et al., 2020). Como respuesta a esta situación y para tratar de que la actividad docente no se interrumpa, las metodologías en línea o mixtas han cogido protagonismo al facilitar un entorno de enseñanza-aprendizaje seguro (Junus et al., 2021). Bajo este contexto, los medios sociales (p.ej., Facebook, Twitter, LinkedIn, Instagram) pueden facilitar la creación de dichos espacios (López-Carril et al., 2021), siendo un recurso pedagógico del que la comunidad docente puede disponer de forma gratuita (Coman et al., 2020; Sobaih et al., 2020).

De entre todos los medios sociales, LinkedIn destaca por su vínculo al ámbito profesional (Tess, 2013), permitiendo que se introduzca en el ámbito educativo para desarrollar competencias profesionales en el alumnado (López-Carril et al., 2020). Además, LinkedIn es un medio social que permite seguir las nuevas tendencias del sector (Marr y DeWaele, 2015), desarrollar la marca personal del alumnado (Zhao, 2021), y apoyar al estudiantado en el proceso de búsqueda de empleo (Carmack y Heiss, 2018). Por estos motivos, la literatura sugiere su inclusión en las clases universitarias (p.ej., López-Carril et al., 2020; Slone y Gaffney, 2016).

Si bien en el contexto de la educación en gestión del deporte destaca el estudio de López-Carril et al. (2021), donde se incorpora con éxito LinkedIn a las clases de gestión del deporte para crear entornos de enseñanzaaprendizaje en línea y mixtos, existe un vacío en la literatura respecto a estudios que clarifiquen las posibilidades de este medio social para crear espacios de aprendizaje en línea. Por ello, el objetivo principal de este trabajo es compartir una innovación educativa, en la cual durante el segundo semestre del curso académico 2019-2020 se introdujo LinkedIn como elemento para crear un entorno de enseñanzaaprendizaje en línea, en las clases de la asignatura troncal de "Gestión y organización de entidades y eventos 
deportivos" (6 créditos ECTS) de tercero de Grado en Ciencias de la Actividad Física y del Deporte (CCAFyD) de la Universitat de València. Un total de 118 estudiantes (72,4\% hombres y 27,6\% mujeres), de dos universidades distintas (Universitat de València y Universidad de Sevilla), participaron en la innovación educativa. De estos, 105 cumplimentaron el cuestionario final (error muestral del 3,19\%, con un intervalo de confianza del 95\%), a partir del que se presentan los resultados de la evaluación de las percepciones del alumnado sobre el material didáctico generado a lo largo de la experiencia educativa.

Por último, con base en el objetivo general de la innovación, se plantea la siguiente pregunta de investigación:

¿Qué percepciones tiene el alumnado de gestión del deporte sobre el uso de LinkedIn para crear entornos de aprendizaje en línea?

\section{Objetivos}

Para realizar el diseño de la innovación, se establecieron dos grandes ámbitos de actuación. Uno vinculado a la persecución de una serie de objetivos específicos vinculados a LinkedIn y el desarrollo del perfil profesional del alumnado, y el otro orientado hacia el trabajo de aspectos incluidos en los contenidos contemplados en la guía docente de la asignatura. A continuación, se procede a enumerar los principales objetivos que guiaron el diseño y desarrollo de la innovación educativa a través de LinkedIn:

2.1. Objetivos vinculados al uso de LinkedIn

2.1.1. Familiarizar a los alumnos con la red profesional LinkedIn, comprender su estructura, y navegar entre sus características esenciales.

2.1.2. Crear un perfil profesional en LinkedIn, incluyendo la selección de un perfil profesional y una foto de portada, la preparación del titular y el DERXW(resumen), así como completar otros apartados del perfil como la experiencia, la formación, las habilidades, las validaciones y las recomendaciones, entre otros aspectos.

2.1.3. Establecer una red profesional de contactos ajustada a los intereses de los estudiantes e incluir al alumnado y al profesorado en el mantenimiento de una relación profesional tras la finalización del curso.

2.1.4. Aprender a identificar los principales grupos de interés de la industria del deporte y cómo dirigirse a ellos.

2.1.5. Desarrollar la marca personal de los alumnos, dándoles herramientas para descubrir qué orientación quieren darle.

2.1.6. Promover la adquisición de competencias digitales en los estudiantes de gestión deportiva.

2.1.7. Fomentar la empleabilidad y el emprendimiento en los estudiantes de gestión deportiva, haciendo hincapié en elementos como la creación de un \&XUFXХP 9 LWHy la búsqueda de empleo o de posibles socios comerciales a través de LinkedIn.

2.2. Objetivos vinculados al desarrollo del temario de la asignatura

2.2.1. Trabajar los siguientes contenidos a través de LinkedIn

- Gestión del PDUNMQJ en las entidades deportivas.

- Innovaciones en el ámbito de las instalaciones deportivas.

- Gestión de los patrocinios en el mundo del deporte.

- El deporte universitario en España.

(c)) BY-NC-ND 2021, Universitat Politècnica de València

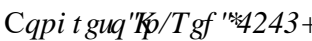


- Las competencias profesionales del gestor del deporte.

- Mujer y deporte.

- Homofobia y deporte.

- Emprendimiento e innovación en la gestión del deporte.

- La gestión del voluntariado en el deporte.

- $\quad \% J \square C D W D$ Inteligencia Artificial y medios sociales en la gestión del deporte.

- Gestión de la marca y relación con los aficionados en la industria del deporte.

- La gestión del deporte en los planes de estudio de CCAFyD.

\section{Desarrollo de la innovación}

La innovación educativa se desarrolló entre los meses de febrero y mayo del 2020, con el apoyo del Vicerrectorado de Ocupación y Programas Formativos de la Universitat de València, a través de las convocatorias de los Proyectos de Innovación Docente (PID) del curso 2019-2020, denominándola "Linkedin (r)evolution: digitalización, emprendimiento y empleabilidad del alumnado de gestión del deporte a través de los medios sociales". A modo de referencia, para plantear el diseño de la innovación se siguieron las recomendaciones específicas para incluir LinkedIn en el contexto universitario, planteadas por López-Carril et al. (2020). Además, se siguió también lo indicado por Manning et al. (2017), quienes recalcan la importancia de impulsar propuestas a través de medios sociales donde el alumnado sea protagonista y aprenda de forma activa. Para ello, se crearon dos grandes ámbitos de actuación en la innovación:

A) El perfil personal del alumnado: destinado a trabajar todos los objetivos recogidos en el anterior subapartado 2.1 .

B) Grupos privados de LinkedIn, donde el alumnado trabajó a través de actividades y debates que el profesorado fue planteando periódicamente para abordar todos los objetivos contemplados en el anterior subapartado 2.2 .

En cuanto al apartado "A", el alumnado recibió una rúbrica de trabajo donde se le proponían una serie de tareas a realizar en su perfil, tanto de forma obligatoria como otras de forma voluntaria. Para apoyar al alumnado en el proceso, el profesorado generó un total de 20 video tutoriales que facilitaron que el alumnado realizase adecuadamente todos los apartados disponibles en su perfil de LinkedIn. Además, se realizaron un total de tres sesiones teóricas. Una presencial y dos en línea síncronas. Por último, también se prestó una atención continua al alumnado a través de mensajes (escritos y audio) en LinkedIn, así como se creó un SRGDWfinal para resolver dudas.

Respecto al apartado "B", se crearon un total de cuatro grupos privados de LinkedIn:

- “\#SMval”: grupo específico para el alumnado del Campus de Blasco Ibáñez de la Universitat de València.

- “\#SMont”: grupo específico para el alumnado del Campus d'Ontinyent de la Universitat de València.

- “\#SMsev": grupo específico para el alumnado de la Universidad de Sevilla.

- "Sport Management Lovers": grupo específico donde converge todo el profesorado y el alumnado participante de la innovación.

En consecuencia, cada estudiante perteneció a dos grupos de la asignatura. Uno específico con el resto de compañeros de clase (\#SMval, \#SMont o \#SMsev) y uno común de todos los estudiantes (Sport Management Lovers). En la Fig. 1 se pueden observar la imagen de perfil y de portada de cada uno de los grupos. En cada uno de estos grupos, el profesorado fue publicando con una periodicidad semanal, una 
propuesta de actividad/debate sobre algún tema vinculado a la gestión del deporte contemplado en el temario de la asignatura. Cada estudiante debía leer la propuesta y participar a través de comentarios, pudiendo interactuar tanto con compañeros de su grupo (en \#SMval, \#SMont o \#SMsev), como con los de otros grupos (en Sport Management Lovers). El profesorado supervisó la evaluación de los debates interviniendo en momentos puntuales para plantear posibles subtemáticas y/o intercambiar puntos de vista con el alumnado.

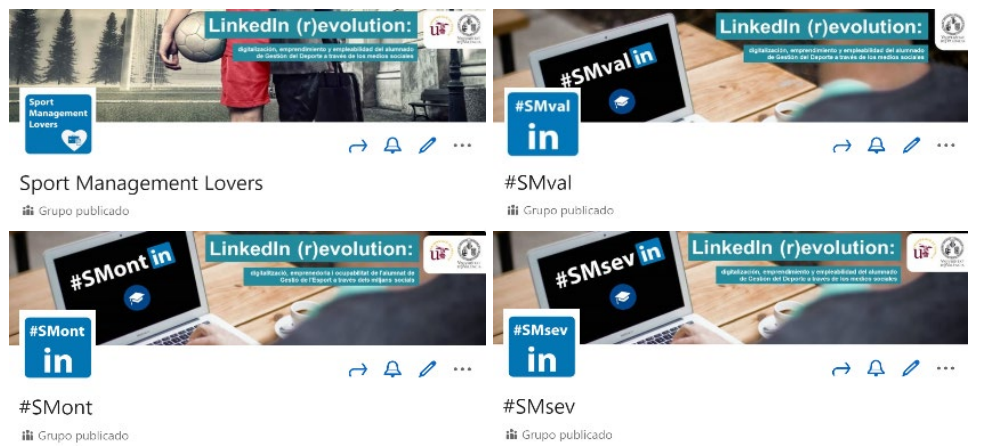

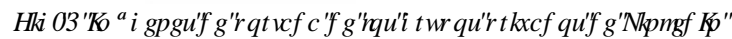

Por otra parte, debido al alto volumen de información y de documentación asociado a la innovación educativa, el profesorado creo una hoja de cálculo Excel en línea a la que se le denominó "Excel mágico". En este, se proporcionaron hipervínculos tanto a todos los perfiles personales del alumnados, como a las publicaciones de cada grupo, materiales de formación, etc. El hipervínculo al Excel mágico se colocó en un lugar visible del espacio de la asignatura en el aula virtual de la Universitat de València.

Respecto al sistema de evaluación y calificación, el alumnado tuvo desde el inicio a su disposición una rúbrica de evaluación (en formato Word), donde fue almacenando las "pruebas" de todo el trabajo realizado a través de hipervínculos. Una vez finalizada la innovación, envió su rúbrica al profesorado de la asignatura con la finalidad de que este pudiese revisar el trabajo realizado. El profesorado revisó cada rúbrica para otorgar una calificación al alumnado de 0 a 10, en función de los criterios de evaluación recogidos en esta. Además, se proporcionó retroalimentación individualizada a cada estudiante a través de un mensaje privado en LinkedIn, donde se le daban indicaciones sobre cómo mejorar su perfil y red de contactos. El peso total de la calificación respecto a la asignatura fue de un $20 \%$ de la nota final de la asignatura.

\section{Resultados}

Para analizar las percepciones del alumnado respecto a los elementos didácticos destacados de la innovación educativa, se les pidió que de forma voluntaria y anónima cumplimentasen un pequeño cuestionario en línea a través de la plataforma LimeSurvey. En este, el alumnado otorgó valoraciones a una batería de enunciados vinculados con la innovación educativa siguiendo una escala del 1 al 10, donde 1 significó la peor puntuación y 10 la máxima. Los resultados se analizaron de forma descriptiva a través del programa estadístico SPSS versión 24.

Respecto a las valoraciones obtenidas (ver Tabla 1), se puede apreciar que la media más elevada se encuentra en el ítem relacionado con la atención prestada por el docente $(\mathrm{M}=9,16 ; \mathrm{DT}=1,38)$ seguida por el ítem que hace referencia al uso del SRGDMpara tratar de resolver dudas $(\mathrm{M}=8,70 ; \mathrm{DT}=1,61)$. Por otro lado, la media más baja aparece en el enunciado relacionado con la valoración de lo aprendido respecto a la gestión deportiva $(\mathrm{M}=7,75 ; \mathrm{DT}=1,96)$. 


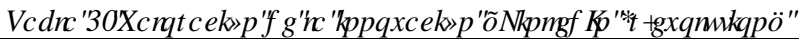

\begin{tabular}{|c|c|c|}
\hline Concepto & Media & DT \\
\hline Valora del 1 al 10 Excel Mágico & 8,26 & 1,91 \\
\hline Los videotutoriales & 8,29 & 1,63 \\
\hline Actividades planteadas en Sport Management Lovers & 8,10 & 1,58 \\
\hline Actividades planteadas en \#SMval, \#SMont y/o \#SMsev & 8,12 & 1,55 \\
\hline Trabajo realizado en vuestro perfil personal & 8,54 & 1,39 \\
\hline Trabajo realizado para construir vuestra red de contactos & 8,25 & 1,47 \\
\hline Sesión teórica de introducción a los medios sociales y a LinkedIn & 8,30 & 1,54 \\
\hline Sesión teórica sobre cómo sacarle el máximo partido a LinkedIn (Realizada por Hugo López) & 8,40 & 1,75 \\
\hline Sesión teórica sobre la generación de contenido en LinkedIn & 8,17 & 1,53 \\
\hline Atención del profesorado a través de mensajes privados en LinkedIn & 9,16 & 1,38 \\
\hline 3RGDMWpara tratar de resolver dudas finales & 8,70 & 1,61 \\
\hline Valoración de lo aprendido en relación a la gestión del deporte & 7,75 & 1,96 \\
\hline Valoración de lo aprendido en relación a la creación de vuestra marca personal & 8,62 & 1,42 \\
\hline Valoración global de la innovación LinkedIn (r)evolution & 8,66 & 1,42 \\
\hline
\end{tabular}

Por último, en relación a la valoración global de la innovación LinkedIn (r)evolution, el alumnado valora muy positivamente la experiencia con una media elevada $(M=8,66 ; \mathrm{DT}=1,42)$, percibiendo que la experiencia les ha ayudado a crear su marca personal $(\mathrm{M}=8,62 ; \mathrm{DT}=1,42)$.

\section{Conclusiones}

LinkedIn se ha mostrado como un recurso pedagógico al alcance del profesorado universitario de gran utilidad a la hora de crear entornos de aprendizajes mixtos o en línea. El alumnado ha valorado de forma positiva la experiencia, otorgando puntuaciones muy altas a todo el material didáctico generado por el equipo docente, y valorando muy positivamente todo el apoyo y retroalimentación recibido a través de los mensajes privados proporcionados por el profesorado en LinkedIn.

Por otra parte, aunque la puntuación media obtenida respecto a lo que el estudiantado percibe que ha aprendido sobre la gestión del deporte sea alta, de cara a futuras experiencias se debe replantear el tipo de actividades propuestas para tratar de conseguir resultados más positivos. En ese sentido, debido a la versatilidad de LinkedIn, se considera que la innovación educativa realizada puede ser trasladada a otros campos de conocimiento con éxito, aunque hasta el momento la mayoría de experiencias recogidas en la literatura provienen del área de la comunicación empresarial, márquetin y negocios (p.ej., Carmack y Heiss, 2018; Florenthal, 2015; Slone y Gaffney, 2016).

En síntesis, después de analizar las percepciones del alumnado respecto a los principales aspectos vinculados a la realización de la innovación educativa, se recomienda que se siga investigando el potencial educativo LinkedIn, dado la importancia que tiene conectar el ámbito profesional con el universitario, así como la situación de pandemia que sigue prolongándose, requiriendo que el profesorado pueda adaptar su docencia a espacios de trabajo en línea como los que puede generar LinkedIn.

\section{Referencias}

ABEZA, G., O'REILLY, N., SÉGUIN, B., y NZINDUKIYIMANA, O. (2015). "Social media scholarship in sport management research: a critical review" en -RXLQDO RI 6SRUWO DQDJHPHQW vol. 26, issue 9, p. 601-618. https://doi.org/10.1123/JSM.2014-0296

ANAGNOSTOPOULOS, C., PARGANAS, P., CHADWICK, S., y FENTON, A. (2018). "Branding in pictures: Using Instagram as a brand management tool in professional team sport organisations" en ( XLRSHDQ6SRUWO DQDJHP HQW 4 XDUALQ, vol. 18, issue 4, p. 413-438. https://doi.org/10.1080/16184742.2017.1410202

CARMACK, H. J., y HEISS, S.N. (2018). "Using the theory of planned behavior to predict college students' intent to use Linkedin for job searches and professional networking" en \&RP P XQIFDURQ6WGIH, vol. 69, issue 2, p. 145-160. https://doi.org/10.1080/10510974.2018.1424003 
CHMAIT, N., WESTERBEEK, H., EIME, R., ROBERTSON, S., SELliTTO, C., y REID, M. (2020). “Tennis influencers: The player effect on social media engagement and demand for tournament attendance" en $\square$ 7DPP DMFVDQG ,QRRP DUFVlvol. 50, art. 101381. https://doi.org/10.1016/j.tele.2020.101381

COMAN, C., ȚÎRU, L. G., MESEȘAN-SCHMITZ, L., STANCIU, C., y BULARCA, M. C. (2020). “Online teaching and learning in higher education during the coronavirus pandemic: Students' perspective" en 6XWDQDEICWW, vol. 12, issue 24, art. 10367. https://doi.org/10.3390/su122410367

DWIDIENAWATI, D., ABDINAGORO, S. B., TJAHJANA, D., GANDASARI, D., y MUNAWAROH. (2020). "Forced shifting to e-learning during the covid-19 outbreak: Information quality, system quality, service quality, and goal orientation influence to e-learning satisfaction and perceived performance" en!, QUHQDURQDO RXLQDORI\$ GDOFHET 7 7HQG IQ \&RPSXU⿴囗 6FHQFH DQG (QJQHUQJ https://doi.org/10.30534/ijatcse/2020/93922020

FILO, K., LOCK, D., Y KARG, A. (2015). "Sport and social media research: A review" en[6SRLWO DQDJ HP HQW HMIHW vol. 18, issue 2, p. 166-181. https://doi.org/10.1016/j.smr.2014.11.001

FLORENTHAL, B. (2015). “Applying uses and gratifications theory to students' LinkedIn usage” en $<R X Q J \square$ \&RQXP HV, vol. 16, issue 1, p., 17-35. https://doi.org/10.1108/YC-12-2013-00416

JUNUS, K., SANTOSO, H.B., PUTRA, P.O.H., GANDHI, A., Y SISWANTINING, T. (2021). "Lecturer Readiness for Online Classes during the Pandemic: A Survey Research" en ( GXFDMRQ 6FIHQFH, vol. 11, issue 3, art. 139. https://doi.org/ 10.3390/educsci11030139

KAPLAN, A. M., Y HAENLEIN, M. (2010). "Users of the world, unite! The challenges and opportunities of social media” en \%XYQHM +RUJRQV, vol. 53, issue 1, p., 59-68. https://doi.org/10.1016/j.bushor.2009.09.003

KOLOKYTHA, E., LOUTROUKI, S., VALSAMIDIS, S., Y FLOROU, G. (2015). "Social media networks as a learning tool" en 3LRFHAD ( FRQRPIFV DQG ) IQDQFH vol. 19, p. 287-295. https://doi.org/10.1016/S2212$\underline{5671(15) 00029-5}$

KOMLJENOVIC, J. (2019). "Linkedin, platforming labour, and the new employability mandate for universities" en[ * REDOWDRQD6RFIHMHDDQF( GXFDMRQVvol. 17, issue 1, p. 28-43. doi.org/10.1080/14767724.2018.1500275

LI, B., DITTMORE, S. W., SCOTT, O. K. M., LO, W.-J., Y STOKOWSKI, S. (2019). "Why we follow: Examining motivational differences in following sport organisations on Twitter and Weibo" en 6SRLWO DQDJH HQWS HIHZ, पQ(3), 335-347. https://doi.org/10.1016/j.smr.2018.04.006

LÓPEZ-CARRIL, S., ANAGNOSTOPOUlOS, C., y PARGANAS, P. (2020). "Social media in sport management

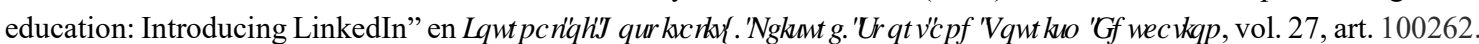
https://doi.org/10.1016/i.jhlste.2020.100262

LÓPEZ-CARRIL, S., VILLAMÓN, M., y AÑÓ, V. (2019). “Conceptualización de los medios sociales: oportunidades para la gestión del deporte” en 5 HRV, vol. 36, p. 578-583. https://doi.org/10.47197/retos.v36i36.68572

LÓPEZ-CARRIL, S., VILLAMÓN, M., y GONZÁLEZ-SERRANO, M.H. (2021). "Linked(In)g sport management education with the sport industry: A preliminary study" en 6XWWQDEICWWvol. 13, issue 4, art. 2275. https://doi.org/10.3390/su13042275

MANCA, S. (2020). "Snapping, pinning, liking or texting: Investigating social media in higher education beyond Facebook" en 7KH, QUAQHWDQG]+LJKHU( GXFDMRQ vol. 44, art. 100707. https://doi.org/10.1016/j.iheduc.2019.100707

MANNING, R., KEIPER, M., y JENNY, S. (2017). "Pedagogical innovations for the millennial sport management student: Socrative and Twitter" en 6SRUW 0 DQDJPHQW ( GXFDURQ -RXLQDOvol. 11, issue 1, p. 45-54. https://doi.org/10.1123/smej.2016-0014

MARR, J., y DEWAELE, C. S. (2015). "Incorporating Twitter within the sport management classroom: Rules and uses

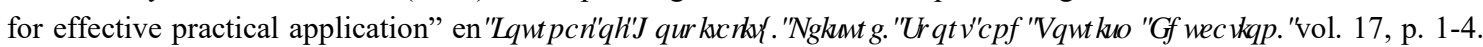
https://doi.org/10.1016/j.jhlste.2015.05.001

(c)) BY-NC-ND 2021, Universitat Politècnica de València

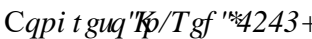


PARGANAS, P., LIASKO, R., y ANAGNOSTOPOULOS, C. (2017). Scoring goals in multiple fields: Social media presence, on-field performance and commercial success in European professional football. Sport, Business and Management, vol. 7, issue 2, p. 197-215. https://doi.org/10.1108/SBM-11-2016-0072

RATTEN, V., y JONES, P. (2020). "Covid-19 and entrepreneurship education: Implications for advancing research and practice" en ,QMHQDURQDO -RXQQDO RID ODQDJPHQW (GXFDURQ art. 100432. https://doi.org/10.1016/j.ijme.2020.100432

SELWYN, N., y STIRLING, E. (2016). "Social media and education... now the dust has settled" en[/ HDQQDआO HAD] DQG77 FFQRQRJ \vol. 41, issue 1, p. 1-5. https://doi.org/10.1080/17439884.2015.1115769

SLONE, A. R., y GAFFNEY, A. L. H. (2016). "Assessing students' use of LinkedIn in a business and professional communication course" en \&RPPXQIFDMRQ 7HDFHU vol. 30, issue 4, p. 206-214. https://doi.org/10.1080/17404622.2016.1219043

SOBAIH, A. E. E., HASANEIN, A. M., y ELNASR, A. E. A. (2020). "Responses to COVID-19 in higher education: Social media usage for sustaining formal academic communication in developing countries" en 6XWDQDDEIOW, vol. 12, issue 16, art. 6520. https://doi.org/10.3390/su12166520

STATISTA (2020). "Number of social network users worldwide from 2017 to 2020". https://www.statista.com/statistics/278414/number-of-worldwide-social-network-users/ [Consulta: 27 de agosto de 2020].

TESS, P.A. (2013). "The role of social media in higher education classes (real and virtual) - A literature review" en \&RP SXWALIQ+ XP DQ\% /KDVIRU vol. 29, issue 5, p. A60-A68. https://doi.org/10.1016/j.chb.2012.12.032

YAN, G., WATANABE, N.M., SHAPIRO, S.L., NARAINE, M.L., Y HULL, K. (2019). "Unfolding the Twitter scene of the 2017 UEFA Champions League Final: social media networks and power dynamics" en (XLRSHDQ 6SRUW 0 DQDJ HP HQW/ XDUसAD, vol. 19, issue 4, p. 419-436. https://doi.org/10.1080/16184742.2018.1517272

ZHAO, X. (2021) “Auditing the "Me Inc.”: Teaching personal branding on LinkedIn through an experiential learning method" en \&RP P XQLFDURQ77HDFHU vol. 35, issue 1, p. 37-42, https://doi.org/10.1080/17404622.2020.1807579 